Nuha Suzaly*1, Marie Christina Keller ${ }^{1}$, Silke Hügl, Elvira Karsten, Thomas Lenarz, Thomas S. Rau

\title{
Characterization of a measurement setup for the thermomechanical characterization of curved shape memory alloy actuators
}

\begin{abstract}
The bend and free recovery (BFR) test according to ASTM F2082 is a standard method to determine the transition temperatures of Nitinol shape memory alloys (SMAs). Unfortunately, this standard method is limited to SMA wires which are straight in its trained shape. Thus, the standard BFR test is not suitable for thermomechanical characterization of curved Nitinol SMA wires which should serve as actuators in cochlear implants in future. We developed a modified BFR measurement setup to determine the active austenite finish $\left(A_{\mathrm{F}}\right)$ temperature of these very thin wires $(\varnothing 100 \mu \mathrm{m})$. The active $A_{\mathrm{F}}$ temperature specifies the completion of the shape recovery upon heating. A parametric study of the measurement setup was carried out to investigate the influence of the heating rate on the observed active $A_{\mathrm{F}}$ temperature and to verify the repeatability of the measurement setup. First, the curved wire was straightened in a cold water bath before inserting it into a water bath that is gradually heated from $5{ }^{\circ} \mathrm{C}$ to $45^{\circ} \mathrm{C}$. The shape change of the previously straightened wire was then recorded throughout the experiment using a digital microscope. Five different heating rates were employed: $0.25 \mathrm{~K} / \mathrm{min}, 0.33 \mathrm{~K} / \mathrm{min}$, $0.5 \mathrm{~K} / \mathrm{min}, 1 \mathrm{~K} / \mathrm{min}$ as well as an unregulated maximum heating rate achievable of approximately $1.5 \mathrm{~K} / \mathrm{min}$. Furthermore, an investigation on the test-retest reliability was performed with three wires by repeating the experiment ten times with each wire. The results of this study revealed no influence of the heating rate on the thermomechanical response of the wires. Based on data from this study, a regulated heating rate of $1 \mathrm{~K} / \mathrm{min}$ is suggested for future investigations, as this reduces the duration of the
\end{abstract}

\footnotetext{
*Corresponding author: Nuha Suzaly: Hannover Medical School, Department of Otolaryngology, Carl-Neuberg-Str. 1, Hanover, Germany, suzaly.nuha@mh-hannover.de Marie Christina Keller: Hannover Medical School, Department of Otolaryngology, Hanover, Germany

Silke Hügl, Thomas Lenarz, Thomas S. Rau: Hannover Medical School, Department of Otolaryngology, Institution and Cluster of Excellence EXC 2177/1 “Hearing4all", Hanover, Germany Elvira Karsten: Leibniz Universität Hannover, Institut für Werkstoffkunde (Materials Science), Garbsen, Germany ${ }^{1}$ both authors contributed equally
}

measurement from four hours to less than an hour. The values obtained from each wire through the test-retest reliability investigation showed a standard deviation of $1.9 \mathrm{~K}$, $1.1 \mathrm{~K}$ and $2.1 \mathrm{~K}$ respectively. Our developed measurement setup demonstrates appropriate repeatability of the measurements.

Keywords: shape memory alloy, thermomechanical characterization, Nitinol, NiTi, bend and free recovery, cochlear implant, shape memory actuator

https://doi.org/10.1515/cdbme-2019-0112

\section{Introduction}

Nitinol (NiTi) is a shape memory alloy (SMA) with the ability to return to a pre-defined shape when heated after being plastically deformed at lower temperatures. Besides the biocompatibility of NiTi, its shape memory effect (SME) makes it attractive for the use as an actuator in medical applications such as in cochlear implants (CIs). CIs are used to treat deafness or severe hearing loss by electrically stimulating the auditory nerve within the inner ear (cochlea). The spatial proximity of the electrode array plays an important role and this may be achieved in various ways such as a pre-shaped geometry of the electrode carrier itself [1] or through actuation [2-4]. Thus, actuation with the help of a NiTi wire trained to a curved shape is proposed here.

Besides being able to train the NiTi wire to a specific geometry, transition temperatures of the material need to be determined. The transition temperatures are temperatures at which the material changes from the martensite state (deformation at lower temperatures) to the austenite state (reverse deformation at higher temperatures). The active austenite finish $\left(A_{\mathrm{F}}\right)$ temperature depicts the completion of the shape recovery upon heating [5], and it is of high interest when utilizing these NiTi wires as an actuator in CIs.

One of the experimental methods used to determine the austenite temperatures is through a standard test method described by the American Society for Testing and Materials 
(ASTM) as the "bend and free recovery" (BFR) test [6]. However, this test is limited to NiTi wires that display a straight trained shape and it is based on contact methods to measure the shape change of the material. Therefore, we developed a measurement setup to determine the active $A_{\mathrm{F}}$ temperature of curved NiTi wires.

\section{Material and Methods}

\subsection{Shape Memory Alloy actuators}

$\mathrm{NiTi}$ wires (hereinafter referred to as 'NiTi inlay') with a diameter of $100 \mu \mathrm{m}$ and a Nickel content of 50.8 at.\% were manufactured (by G.RAU GmbH \& Co. KG, Pforzheim, Germany) and trained to a two-dimensional curved shape shown in Figure 1. This shape was derived from a specific human cochlea geometry by a manual microgrinding technique followed by an image registration and segmentation process [7-8].

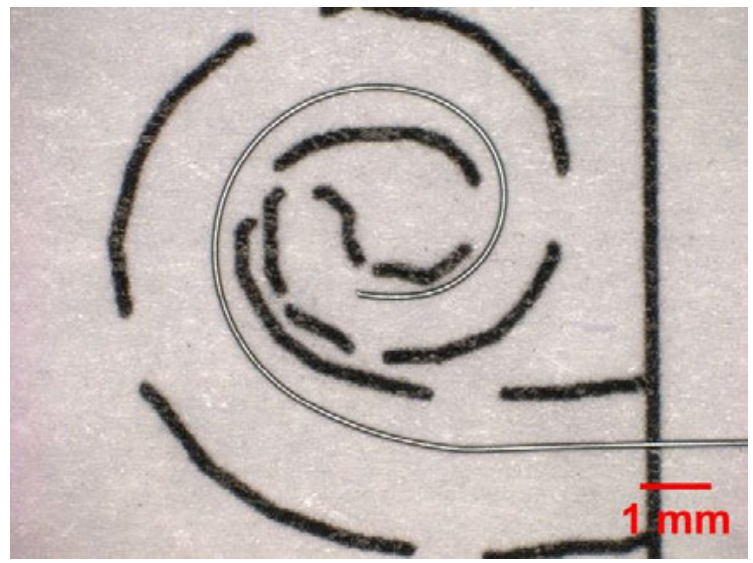

Figure 1: Image of $\mathrm{NiTi}$ inlay with a sketch of the cochlea geometry in the background

According to the differential scanning calorimetry (DSC) received from the manufacturer, the austenite start $\left(A_{\mathrm{S}}\right)$ and $A_{\mathrm{F}}$ temperatures of the NiTi inlays are $29.9^{\circ} \mathrm{C}$ and $32.4{ }^{\circ} \mathrm{C}$ correspondingly.

\subsection{Measurement setup and procedure}

A measurement setup based on the standard BFR test [6] and inspired by the work of Undisz et al. [9] was developed to determine the active $A_{\mathrm{F}}$ temperature of the NiTi inlays. Due to the geometry and size of the NiTi inlays, a non-contact visual method was used for the measurement setup.
The setup (Figure 2) consists of a water bath tank which is equipped with a digital heating thermostat (LAUDA ET 20G, Lauda Dr. R Wobser GmbH \& Co. KG, LaudaKönigshofen, Germany), a digital microscope camera (DinoLite), a thermocouple (OMEGA Engineering, Mini Hypodermic Probes, HYP1-30-1/2-T-G-60-SMPW-M) for temperature acquisition near the NiTi inlay and a 3D-printed shaft to mount the NiTi inlay holder used to fixate the NiTi inlay. Furthermore, a measurement grid served as an image background.

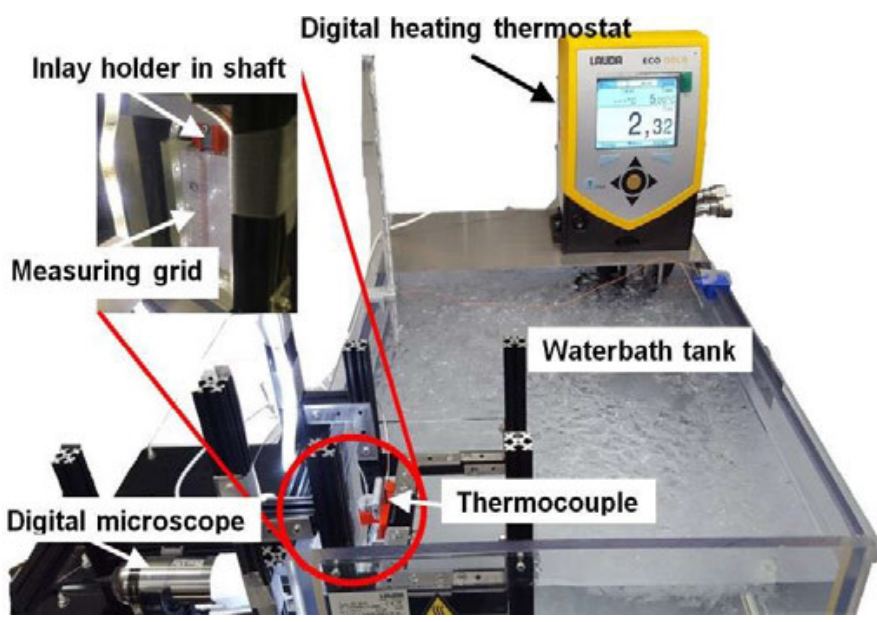

Figure 2: Measurement setup for the thermomechanical characterization of the NiTi inlays

After fixating and aligning the NiTi inlay to the inlay holder, it was manually straightened using lab tweezers in a separate cold water bath that is at least $2^{\circ} \mathrm{C}$. The straightened NiTi inlay is then mounted to the shaft inside the water bath tank. The setup design allows the NiTi inlay to freely move once it is placed inside the water bath. The water bath tank had an initial temperature of at least $4.5^{\circ} \mathrm{C}$.

Using the digital heating thermostat, the water bath was then heated from $4.5^{\circ} \mathrm{C}$ to $45^{\circ} \mathrm{C}$ gradually with a constant predefined heating rate. Simultaneously to the heating of the water bath, the shape change of the NiTi inlay was recorded using a digital microscope camera with an image acquisition rate of 20 images per Kelvin. The temperature near the NiTi inlay was continuously recorded using a thermocouple during the experiment. The $A_{\mathrm{F}}$ temperature of the NiTi inlay is then determined subjectively by scanning the sorted images according to the recorded temperatures until no further shape change is visible in the acquired images. 


\subsection{Thermomechanical characterization}

To investigate the influence of the heating rate on the determination of the transition temperatures, five different heating rates were carried out: $0.25 \mathrm{~K} / \mathrm{min}, 0.33 \mathrm{~K} / \mathrm{min}$, $0.5 \mathrm{~K} / \mathrm{min}, 1 \mathrm{~K} / \mathrm{min}$. The maximum heating rate achievable with the digital thermostat (later determined to be approximately $1.5 \mathrm{~K} / \mathrm{min}$ ) was also explored. Each heating rate was investigated using different NiTi inlays of the same material properties and batch. Additionally, two of the investigated $\mathrm{NiTi}$ inlays were heated at the maximum controlled heating rate of $1 \mathrm{~K} / \mathrm{min}$.

After investigating the influence of the heat rate, an evaluation on the test-retest reliability was carried out by performing the experiment ten times with three NiTi inlays. The heating rate for this investigation was set to $1 \mathrm{~K} / \mathrm{min}$ based on the results obtained from the previous experiments carried out with NiTi inlays at different heating rates.

\section{Results}

\subsection{Measurement setup}

A non-contact method to record the shape change of the NiTi inlays between $4.5^{\circ} \mathrm{C}$ and $45^{\circ} \mathrm{C}$ was able to be achieved with measurement setup developed. All NiTi inlays were able to be successfully tested using the measurement setup developed. The straightening of the NiTi inlays in the cold water bath was also possible.

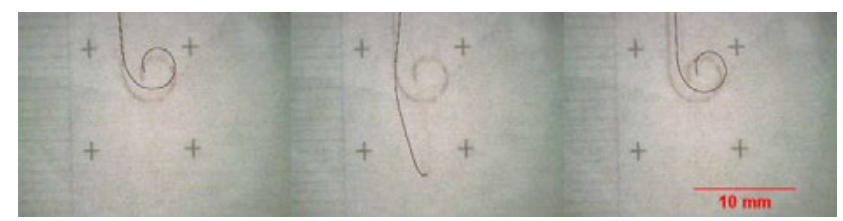

Figure 3: Recorded image of NiTi inlay before straightening at $5{ }^{\circ} \mathrm{C}$, after straightening and at $45^{\circ} \mathrm{C}$ after shape recovery (from left to right)

However, difficulty in achieving a consistent homogenous straightening of the NiTi inlay was observed. Although the condition of the NiTi inlay varied after straightening, this did not affect the ability of the NiTi inlay to return to its trained geometry as seen in Figure 3. Due to the water current generated by the water pump of the digital heating thermostat, motion displacement of the freely hanging NiTi inlay was seen between the images acquired.

\subsection{Influence of heating rate}

The achieved active $A_{\mathrm{F}}$ temperatures at different heating rates were observed (Figure 4 ). No visible pattern or correlation between the heating rates and the determined active $A_{\mathrm{F}}$ was seen.

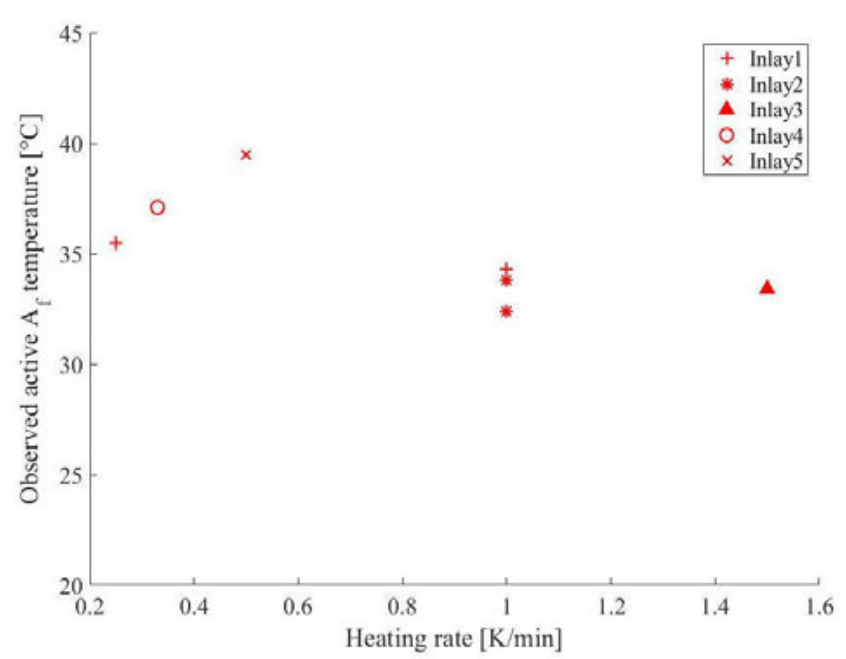

Figure 4: Observed active $A_{F}$ temperatures for the different heating rates investigated

The difference in the active $A_{\mathrm{F}}$ temperatures observed between two different heating rates for the same NiTi inlay (depicted as Inlay 1 in Fig. 4) is $1 \mathrm{~K}$. The same difference was also seen by heating a NiTi inlay twice at the same heating rate. Due to the results achieved, the maximum controlled heating rate of $1 \mathrm{~K} / \mathrm{min}$ was chosen for all further experiments.

\subsection{Test-retest reliability}

Results obtained from the earlier investigation were taken into consideration in the experiments to investigate the testretest reliability. This study revealed the following results shown in Figure 5. 


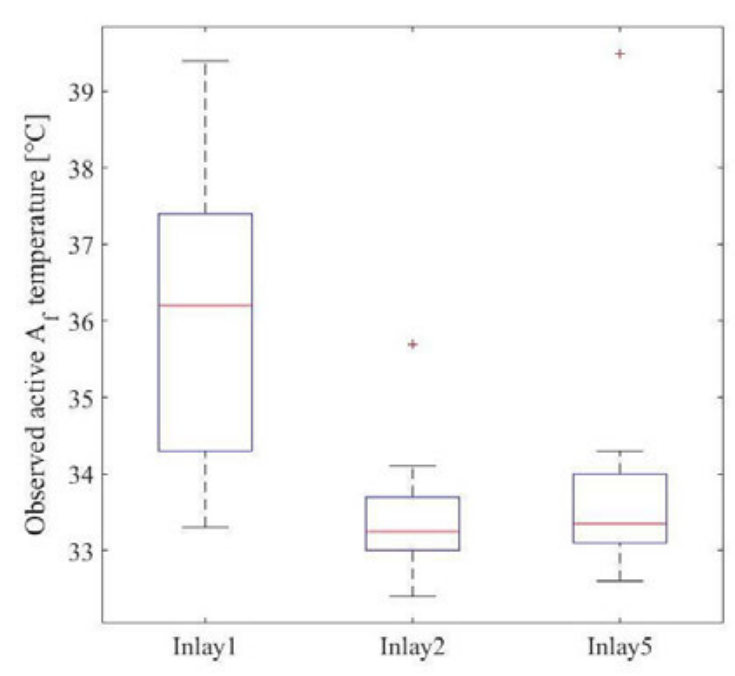

Figure 5: Observed active $A_{F}$ temperatures for three $\mathrm{NiTi}$ inlays measured ten times each

The average $A_{\mathrm{F}}$ temperature values obtained for each NiTi inlay were $36.0 \pm 1.9 \mathrm{~K}, 33.7 \pm 1.1 \mathrm{~K}$ and $34.1 \pm 2.1 \mathrm{~K}$, correspondingly. The difference between the maximum and minimum active $A_{\mathrm{F}}$ temperature observed over all experiments performed within this study is $7.5 \mathrm{~K}$.

\section{Discussion}

We developed a non-contact visual measurement setup within the workgroup to determine the active $A_{\mathrm{F}}$ temperature of curved NiTi inlays. The NiTi inlays were trained to a curved shape that was derived from a specific human cochlea geometry. The thermomechanical process was performed by the manufacturer in a way that the wires return to its trained shape after deformation when exposing the wire to temperatures above $32{ }^{\circ} \mathrm{C}$. To verify this, a measurement setup based on the BFR test was developed [6,9]. This parametric study to characterize the measurement setup showed that further improvement on the straightening of the $\mathrm{NiTi}$ at lower temperatures below $5{ }^{\circ} \mathrm{C}$ is needed. This is to ensure that the NiTi inlays display a uniform straightened shape within all experiments carried out. Reduction of the water current within the water bath tank also needs to be considered to obtain images that only show the shape change of the NiTi inlays.

Changing the heating rate did not show any visible trend, which can be explained by the non-uniform straightening of the NiTi inlays within the experiments performed. The active $A_{\mathrm{F}}$ temperatures observed using the developed measurement setup were higher than the $A_{\mathrm{F}}$ temperature specified in the
DSC curve from the manufacturer. This can be explained by the data interpretation made between both methods to determine the $A_{\mathrm{F}}$ temperature. Therefore, the $A_{\mathrm{F}}$ value in the DSC curve only provides orientation to the scope in which the $A_{\mathrm{F}}$ temperature of the NiTi inlays can lie. With the use of the developed measurement setup, it is also possible to determine the active $A_{\mathrm{F}}$ temperature that is more realistic for the desired clinical application.

\section{Author Statement}

Research funding: The presented work was funded by the German Research Association (DFG, RA 2751/1-1, Cluster of Excellence EXC 2177/1 "Hearing4all"). Conflict of interest: Authors state no conflict of interest

\section{References}

[1] Nagy R, Jarabin JA, Perényi Á, Dimák B, Tóth F, Jóri ;, Kiss JG, Rovó L. Long-term hearing preservation with slimperimodiolar $\mathrm{Cl} 532 \circledast$ Cochlear Implant Array. American Journal of Otolaryngology and Head and Neck Surgery 2018; Issue 4; Article 1019; 1-6.

[2] Stieghorst J, Doll T. Dispersed hydrogel actuator for modiolar hugging cochlear implant electrode arrays. IEEE Trans Biomed Eng 2016; 63:294-300.

[3] Zentner L, Griebel S, Wystup C, Hügl S, Rau TS, Majdani O. Synthesis process of a compliant fluidmechanical actuator for use as an adaptive electrode carrier for cochlear implants. Mech Mach Theory 2017; 112:155-71.

[4] Rau TS, Hügl S, Lenarz T, Majdani O. Toward steerable electrodes: anoverview of concepts and current research. In Current Directions in Biomedical Engineering 2017;3(2):765769.

[5] ASTM F2005-05, Standard terminology for nickel-titanium shape memory alloys, ASTM International, West Conshohocken, PA, 2005.

[6] ASTM F2082-06, Standard test method for determination of transformation temperature of nickel-titanium Shape Memory Alloys by Bend and Free Recovery, ASTM International, West Conshohocken, PA, 2006.

[7] Rau TS, Würfel W, Lenarz T, Majdani O. Three-dimensional histological specimen preparation for accurate imaging and spatial reconstruction of the middle and inner ear. International journal of computer-assisted radiology and surgery 2013; 8(4):481-509.

[8] Hügl S, Eckardt F, Lexow GJ, Majdani O, Lenarz T, Rau TS.Increasing the resolution of morphological 3D image data sets through image stitiching: application to the temporal bone. Computer Methods in biomechanics and Biomedical Engineering: Imaging \& Visualization 2017; 5(6):438-445

[9] Undisz A, Fink M, Rettenmayr M. Determination of transformation properties of thin medical grade NiTi- wire by High Resolution Bend and Free Recovery Testing. Journal of Materials Engineering and Performance 2009; 18(5-6):814817 